\title{
Influence of the crustal magnetic field on the Mars aurora electron flux and UV brightness
}

\author{
D.V. Bisikalo ${ }^{a}$, V.I. Shematovich ${ }^{\mathrm{a}, *}$, J.-C. Gérard ${ }^{\mathrm{b}}$, B. Hubert ${ }^{\mathrm{b}}$ \\ a Institute of Astronomy of the Russian Academy of Sciences, Pyatnitskaya street, 48, 119017 Moscow, Russia \\ ${ }^{\mathrm{b}}$ Laboratoire de Physique Atmosphérique et Planétaire, STAR Institute, Université de Liège, Allée du 6 Août, 19C, B-4000 Liège, Belgium
}

\section{A R T I C L E I N F O}

\section{Article history:}

Received 9 March 2016

Revised 7 July 2016

Accepted 29 August 2016

Available online 16 September 2016

\section{Keywords:}

Mars

Aurorae

Ultraviolet observations

Magnetic fields

\begin{abstract}
A B S T R A C T
Observations with the SPICAM instrument on board Mars Express have shown the occasional presence of localized ultraviolet nightside emissions associated with enhanced energetic electron fluxes. These features generally occur in regions with significant radial crustal magnetic field. We use a Monte-Carlo electron transport model to investigate the role of the magnetic field on the downward and upward electron fluxes, the brightness and the emitted power of auroral emissions. Simulations based on an ASPERA-3 measured auroral electron precipitation indicate that magnetic mirroring leads to an intensification of the energy flux carried by upward moving electrons- from about $20 \%$ in the absence of crustal magnetic field up to $33-78 \%$ when magnetic field is included depending on magnetic field topology. Conservation of the particle flux in a flux tube implies that the presence of the B-field does not appreciably modify the emission rate profiles for an initially isotropic pitch angle distribution. However, we find that crustal magnetic field results in increase of the upward electron flux, and, consequently, in reduction of the total auroral brightness for given energy flux of precipitating electrons.
\end{abstract}

(C) 2016 Elsevier Inc. All rights reserved.

\section{Introduction}

The first measurements of magnetic field on Mars by Acuña et al. (1998) were based on the magnetometer measurements on board the Mars Global Surveyor (MGS) mission. They confirmed the lack of a global magnetic field by Mariner 5, Mars 2, 3 and 5 and Mars Phobo-2 spacecraft but revealed the presence of strong localized residual crustal magnetic field concentrated below the southern hemisphere highlands. Analysis of the nightside measurements with the Electron Reflectometer (ER) instrument by Mitchell et al. (2001) implied the existence of "magnetocylinders" marked by series of plasma voids separating electron flux spikes. These electron spike events are concentrated in regions of relatively strong radial component of the residual magnetic field showing the energy spectra similar to the magnetosheath electrons. The measurements were interpreted as evidence of past or present reconnection of the residual magnetic field lines to the interplanetary magnetic field lines.

The interest for auroral precipitation into the Martian atmosphere was triggered by the discovery of the Martian far ultraviolet aurora by Bertaux et al. (2005). The first evidence for precipitation of energetic electrons was provided by Brain et al. (2006) who

\footnotetext{
* Corresponding author: Fax: +7(495)9515557.

E-mail addresses: bisikalo@inasan.ru (D.V. Bisikalo), shematov@inasan.ru (V.I. Shematovich).
}

detected the presence of peaked electron distributions measured near $400 \mathrm{~km}$ with the ER instrument on board MGS. These observations revealed hundreds of auroral-like events characterized by energized electrons with a peak energy ranging from $\sim 200 \mathrm{eV}$ to $4 \mathrm{keV}$. Concomitant perturbations of the horizontal magnetic field components were measured and found to be consistent with fieldaligned currents density comparable with terrestrial field-aligned currents. These events correspond to auroral-like energized electron population in crustal field cusp regions, near a field-aligned current region and the boundary between open and closed field lines. Brain et al. (2006) suggested that they occur on magnetic field lines connecting the shocked solar wind to crustal magnetic fields. They pointed out that downward going electrons between $100 \mathrm{eV}$ and $1 \mathrm{keV}$ are generally nearly isotropic. At the same time downward-traveling sheath electrons on the Martian nightside span a wide range of pitch angle distributions (PADs). Brain et al. (2007) analyzed the distributions of electron PADs measured by MGS interpreting observed PADs as indicators of magnetic field topology near Mars. Also, Lillis et al. (2011) investigated the threedimensional distribution of ionization from precipitating sheath electrons on the Martian nightside, and showed the average PADs of downward-traveling electrons in five sample locations, pointing out that most are in fact non-isotropic (the only one which is isotropic has almost no flux). The most energetic events were preferentially observed during periods of disturbed solar wind conditions such as passing coronal mass ejections. Among the three 
types of accelerated electron events identified by Halekas et al. (2008), localized events occur in strong magnetic cusp regions and may be associated with signatures of field-aligned currents.

Measurements made with the Analyzer of Space Plasma and Energetic Atoms (ASPERA-3) set of plasma instruments on Mars Express (Barabash et al., 2006) also indicated that regions of open magnetic field are often present near strong and moderate crustal fields on the Martian nightside (Lundin et al., 2006a, 2006b). The associated electrons generally showed fluxes several orders of magnitude higher than elsewhere on the nightside, with an inverted-V energy distribution peaking near hundred eV's, similar in shape to the more energetic inverted-V events generally measured within terrestrial auroral arcs. Lundin et al. (2006b) pointed out that these electron distributions bear the signature of downward acceleration in an upward electric field.

The role of crustal magnetic fields on electron precipitation and ionization was modeled by Lillis et al. (2009) using a kinetic Monte Carlo electron transport model. In particular, they examined the effects of the crustal B-field on the volume ionization rate and found that the peak values decrease with increasing $B$ intensity by less than $20 \%$ below $200 \mathrm{~km}$ in the case of a measured initial electron energy spectrum with an isotropic pitch angle distribution (PAD). The drop of the ionization rate versus the magnetic field intensity was more pronounced in the case of a trapped electron population, but nearly doubled for a beamed pitch angle distribution in a field of several tens of nT. Lillis and Fang (2015) examined the effects on ionization profiles and total integrated ionization for a wide range of initial electron pitch angles and energies and crustal field strengths.

Lillis and Brain (2013) made a thorough study of the downward-traveling superthermal electrons measured by MGS near $400 \mathrm{~km}$ on the nightside. They showed that a more horizontal orientation of the magnetic field delays electron access to the atmosphere and that the highest precipitating fluxes occur for negligible crustal field magnitude and the most vertical crustal field orientation. When the B-field elevation angle is in the range $60^{\circ}-90^{\circ}$, the precipitated electron flux moderately increases in regions of larger solar wind proxy pressure. Lillis et al. (2011) and Lillis and Brain (2013) pointed out that the strength and topology of the crustal magnetic field influence the precipitation pattern. This variation of the strength and geographic pattern of the shielding effect of Mars' crustal field exemplifies the complex interaction between this field and the solar wind. The energy distribution of energetic electrons in the Mars nightside over strong crustal field regions was further investigated by Shane et al. (2016). They found that, when solar zenith angle exceeds $110^{\circ}$, energy deposition mainly occurs along vertical field lines. They showed that most of the precipitated electrons have an energy of $15-30 \mathrm{eV}$ and are magnetically reflected or backscattered, so that a fraction of less than $16 \%$ deposit their energy in the Martian atmosphere.

The first detection of an ultraviolet aurora was made at the limb with the Spectroscopy for Investigation of Characteristics of the Atmosphere of Mars (SPICAM) UV spectrograph on board Mars Express (Bertaux et al., 2005a). Further observations were obtained in the nadir direction in regions having a crustal magnetic field by Leblanc et al. (2008). Recently, Gérard et al. (2015) searched the full SPICAM database to identify signatures of CO Cameron and $\mathrm{CO}_{2}{ }^{+}$doublet ultraviolet auroral emissions and analyze concurrent ASPERA-3/ELS measurements. Spectral signatures of auroral emissions were found in the vicinity of the boundary region between open and closed field lines. The mean electron energy measured at the precipitation maximum ranged from 150 to $280 \mathrm{eV}$. The low number of MUV auroral detections with SPICAM indicates that the Mars aurora is a time dependent feature. Recently, the diffuse aurora at Mars was discovered (Schneider et al., 2015) in the observations by the IUVS instrument onboard NASA MAVEN spacecraft.
Actually, MAVEN IUVS instrument has observed much more often the auroral events than MEX SPICAM instrument, therefore aurora database for Mars soon will be extended. Soret et al. (2016) found no proportionality between electron fluxes measured in the upper thermosphere and nadir auroral intensity. They used a Monte Carlo electron transport model to simulate auroral emissions based on monoenergetic electron precipitation and two energy spectra measured with ASPERA-3/ELS complemented by simultaneous nadir SPICAM observations. They calculated the peak altitudes in the vicinity of $135 \mathrm{~km}$, in good agreement with the SPICAM limb observations. Their predicted vertically integrated intensities for several emission features were generally overestimated, possibly as a consequence of 3-D magnetic field topology and electron mirroring not being accounted for in their model.

In this study, we examine the effects of the presence of the vertical component of crustal magnetic field on the upward and downward propagating electron fluxes and on the auroral emission rate of the $\mathrm{CO}$ Cameron and $\mathrm{CO}_{2}{ }^{+}$bands. For this purpose, we introduce magnetic mirroring in the Monte Carlo simulations, under the constraint of the first adiabatic invariant. We then compare the upward $F_{\text {up }}$ and downward $F_{\text {down }}$ electron energy distribution with and without a vertical $B$-field. We show how the $F_{\text {up }} / F_{\text {down }}$ flux ratio varies with altitude as a function of parameters describing the vertical structure of the field. Finally, we examine the influence of the magnetic field structure on the vertical profile and the emitted power of the auroral emissions.

\section{The model}

\subsection{Monte Carlo model description}

In this study, the influence of the residual crustal magnetic field on the excitation processes leading to the aurora emissions is analyzed using simulations realized with a model of electron transport in the Martian thermosphere. This model is based on a kinetic Monte Carlo approach. It was described by Shematovich et al. (2008) for the Mars dayglow and applied by Gérard et al. (2008) to the Venus dayglow, by Soret et al. (2016) to the Mars aurora and by Shematovich et al. (2011) to proton and $\mathrm{H}$ atom precipitation into the Martian atmosphere.

Energetic electrons from the induced Martian magnetosphere can precipitate and interact with the atmosphere where they can lose their kinetic energy in elastic, inelastic and ionization collisions with the ambient atmospheric gas. The energy loss of the precipitating electrons is calculated by the kinetic Boltzmann equation (Shematovich et al., 2008), involving transport of electrons, production of primary and secondary electrons and elastic and inelastic scattering terms. The kinetic Monte Carlo method is an efficient approach to solve kinetic Boltzmann equations for atmospheric systems in the stochastic approximation, when the collisions of all electrons in each cell and electron transport are considered separately at each time step in contrast with the test-particle approach (Lillis et al., 2009, 2011). It calculates the system evolution from the initial to the steady state, from $300 \mathrm{~km}$ to $75 \mathrm{~km}$. The outputs are vertical emission profiles for various $\mathrm{CO}$, $\mathrm{CO}_{2}{ }^{+}$and $\mathrm{O}$ emissions. More details about the kinetic Monte Carlo model can be found in Shematovich et al. (2008).

The neutral atmosphere is taken from outputs of the Mars Global Ionosphere-Thermosphere Model (M-GITM) (Bougher et al., 2015) for a solar longitude of $0^{\circ}$, a latitude of $50^{\circ}$ South, a longitude of $180^{\circ}$ (which correspond to a region with significant residual magnetic field on Mars), a F10.7 index of 30 at Mars (average value at the time of the auroral detections) and at midnight local time. The M-GITM takes into account the fundamental physical parameters, ion-neutral chemistry, and key radiative processes of Mars from the ground to the exosphere $(0-300 \mathrm{~km})$. 
The processes which govern the relative importance of the collisions and energy loss of the electrons were listed in Shematovich et al. (2008). The cross sections for electron impact dissociative excitation and ionization of the $\mathrm{CO}_{2}$ states and their analytical expression are taken from the compilation by Shirai et al. (2001). The excitation cross sections of the $\mathrm{CO}\left(\mathrm{a}^{3} \Pi\right)$ state by electron impact on $\mathrm{CO}_{2}$ is still largely uncertain. It was initially measured by Ajello (1971) and was re-evaluated several times (Erdman and Zipf, 1983; Avakyan, 1998). It was recently corrected to account for the determination of the $\mathrm{CO}\left(\mathrm{a}^{3} \Pi\right)$ radiative lifetime by Gilijamse et al. (2007). It leads to the value of $8 \times 10^{-17} \mathrm{~cm}^{2}$ at $80 \mathrm{eV}$ adopted in this study, with an uncertainty of about 25\% (Gronoff et al., 2012). A second source of $\mathrm{CO}\left(\mathrm{a}^{3} \Pi\right)$ state is the direct excitation by electrons impact on $\mathrm{CO}$. The corresponding excitation cross section is taken from Shirai et al. (2001). The excitation cross section of the $\mathrm{CO}_{2}{ }^{+}$Fox-Duffendack-Barker (FDB) bands, which correspond to the $\mathrm{A}^{2} \Pi_{\mathrm{u}} \rightarrow \mathrm{X}^{2} \Sigma_{\mathrm{g}}$ transition of $\mathrm{CO}_{2}{ }^{+}$, was also taken from the compilation of Shirai et al. (2001).

The Monte Carlo model of electron transport rests upon several assumptions. First, it is assumed that the ambient atmospheric gas is characterized by the local Maxwellian velocity distribution functions. Electrons are assumed to precipitate along the crustal magnetic field lines that are considered to be perpendicular to the surface of the planet, therefore, our studies are specific to the vertical or quasi-vertical case. The initial pitch angle distribution (PAD) is assumed to be quasi-isotropic in the sense defined by Decker et al. (1996), i.e. uniform in $\cos ^{2}(\theta)$.

\subsection{Magnetic field model}

The vertical structure of the magnetic field is similar to the approach used by Lillis et al. (2009) in their analysis of the influence of the crustal field on the electron pitch angle distribution (PAD). Since their gyro radius is less than a few kilometers below $1 \mathrm{keV}$ for the Martian B-field intensity, we consider the electrons as bound to a single magnetic field line. We also restrict our analysis to the case of a vertical field line, perpendicular to the atmospheric layers of the Monte Carlo model. The B-field is assumed to decrease with distance above the crustal source according to:

$B(z)=B_{\text {ambient }}+B_{\text {crust, } 100 \mathrm{~km}} \times[100 \mathrm{~km} /(z+a)]^{k}$,

where $z$ is the altitude, $\mathrm{a}$ - the depth of the crustal field source (assumed to be $15 \mathrm{~km}$ beneath the surface), $B_{\text {crust, }} 100 \mathrm{~km}$ is the value of the field at $100 \mathrm{~km}$. The $k$ exponent is a parameter allowed to vary between 2 and 3 . Case $k=2$ would be appropriate for an infinite line of dipoles, $k=3$ - for a single dipole, therefore $k=2.5$ is a reasonable middle ground. In the sample simulations presented here, we use $k=2.5, B_{\text {crust, } 100 \mathrm{~km}}=23 \mathrm{nT}$ (Lillis et al., 2009). A constant ambient magnetic field $B_{\text {ambient }}$ was taken equal to $12 \mathrm{nT}-$ a typical value of the magnetotail field (Ferguson et al., 2005). We also consider the cases with $B_{\text {ambient }}=1.2$, and $0.12 \mathrm{nT}$ to examine how strongly this parameter changes the results of our simulations.

The effect of the ambient field on the mirroring can be understood considering the value of the mirror force. This force is obtained by averaging the magnetic force over one gyration of the moving charged particle. The magnetic moment associated with the current due to the gyration of a charged particle can be written as

$\vec{m}=-\frac{W_{\perp}}{B^{2}} \vec{B}=-\frac{\frac{1}{2} M v_{\perp}^{2}}{B^{2}} \vec{B}$

Where $W_{\perp}$ is the contribution of the perpendicular components of the velocity to the kinetic energy. The mirror force is obtained by averaging the magnetic force exerted on the gyrating particle over one gyration, assuming that the horizontal components of the field are small, which gives (Bittencourt, 2004)

$$
\left\langle\vec{F}_{\triangleleft}\right\rangle=(\vec{m} \cdot \vec{\nabla}) B \vec{e}_{z}=-\frac{|\vec{m}|}{B}[(\vec{B} \cdot \vec{\nabla}) \vec{B}]_{\triangleleft} .
$$

So, if we write the field as a crustal field variable versus $\mathrm{z}$ plus a constant ambient field, we have

$\vec{B}=\vec{B}_{a}+\vec{B}_{c}(z)=\left(B_{a}+B_{c}(z)\right) \vec{e}_{z}$.

Replacing the magnetic field by its value in the expression of the mirror force and of the magnetic moment, it is found that

$\left\langle\vec{F}_{\triangleleft}\right\rangle=-\frac{W_{\perp}}{\left(B_{a}+B_{c}(z)\right)} \frac{\partial B_{c}(z)}{\partial z} \vec{e}_{z}$

Consequently, adding a non-zero, positive, constant ambient field $B_{\mathrm{a}}$ reduces the magnitude of the mirror force, for a given value of the orthogonal part of the kinetic energy $W_{\perp}$. This result can be understood as adding the constant $B_{\text {a }}$ reduces the divergent aspect of the magnetic field lines. The field thus becomes more homogeneous and the mirror effect is reduced, although it may seem paradoxical when the magnitude of the total field is increased.

We assume that the electrons have been accelerated above the upper boundary of the Monte Carlo model so that the accelerating electrostatic potential within the model domain is set to zero. The field line concentration is taken into account as well. Such concentration does not affect the up/down ratios of flux but it will affect the absolute flux numbers.

\section{Results}

To study the influence of the crustal magnetic field on the precipitation of the electrons into the Mars atmosphere we have conducted several simulations using the model described above. To make runs rooted in actual observations, we set the input flux to the measured fluxes of precipitating electrons in the atmosphere of Mars obtained by the ASPERA-3 on board Mars Express. For the simulations we used the energy distribution function of electrons, measured on 10 May 2010 at 07:44 UT at the altitude of $590 \mathrm{~km}$ (Gérard et al., 2015). The energy distribution of the downward moving electrons is presented in Fig. 1. The energy flux strongly decreases above $200 \mathrm{eV}$, reaching a very low value at $300 \mathrm{eV}$ The peak energy is $90 \mathrm{eV}$, the mean electron energy is $\sim 140 \mathrm{eV}$, and the downward energy flux is equal 266 to $1.4 \mathrm{mWm}^{-2}$ (Gérard et al., 2015).

\subsection{Effect on upward and downward electron fluxes}

The number (a) and energy (b) electron fluxes at $286 \mathrm{~km}$ are shown in Fig. 2 for upward direction (solid line - with crustal magnetic field $B_{\text {crust, } 100 \mathrm{~km}}=23 \mathrm{nT}$, and dashed line - without, $B_{\text {crust, } 100 \mathrm{~km}}=0 \mathrm{nT}$ ). The energy flux for downward direction at $286 \mathrm{~km}$ coincides with the ASPERA (Fig. 1). Calculated height profiles of the downward (solid lines) and upward (dashed lines) energy fluxes for these two runs are given in Fig. 3. It is assumed that the crustal magnetic field may be characterized by following typical parameters: $a=15 \mathrm{~km}, k=2.5$, and $B_{\text {ambient }}=12 \mathrm{nT}$. The simulation results obtained in this case indicate that the upward energy flux can be as large as 33\% of the downward one. It is important to note that the formation of the thermal core of the upward flux at low energies is a consequence of the important role played by collisional spreading in the formation of the upward electron flux. In contrast, the upward flux at high energy is mostly due to magnetic mirroring. In case of $B_{\text {crust, } 100 \mathrm{~km}}=0 \mathrm{nT}$, there is no contribution from the magnetic mirroring, which results in a conspicuous reduction of the upward number flux above $\sim 10 \mathrm{eV}$. This leads to a significant decrease, up to $20 \%$, in the upward energy flux. Thus, from comparison of the calculations presented in Figs. 2 and 3 it 


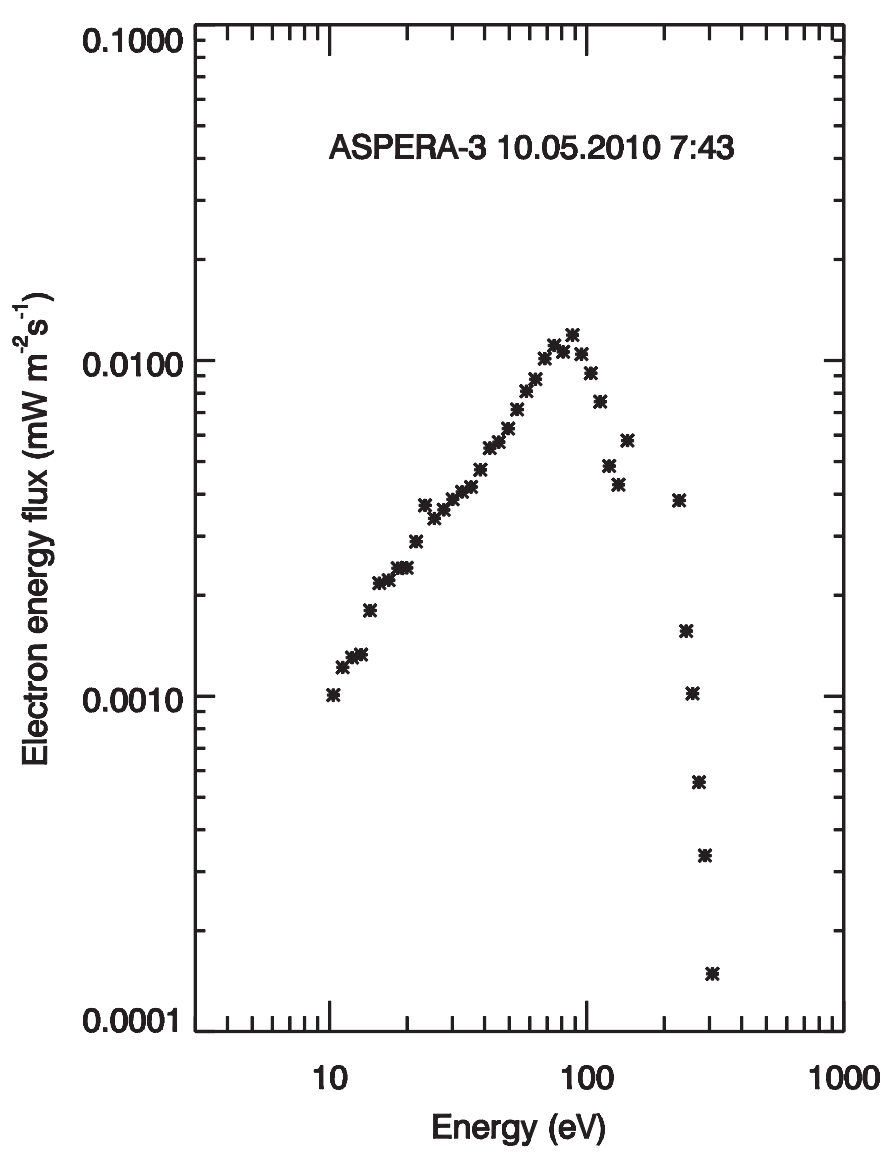

Fig. 1. Spectrum of the downward electron energy flux, measured $590 \mathrm{~km}$ on May 10, 2010 at 7:43 by ASPERA-3 instrument onboard Mars-Express.

can be concluded that the effect of the crustal magnetic field leads to a marked, energy-dependent, increase of the upward electron energy flux from $20 \%$ to $33 \%$.

Several sensitivity runs were conducted in order to analyze how the crustal magnetic field on Mars influences the auroral electron flux and UV brightness. It is naturally expected that the most important factor influencing magnetic mirroring and hence the solution, is the topology of the magnetic field (Lillis and Fang, 2015). Given that this topology of the magnetic field is not well known, we have conducted calculations for three different values of the $k$ parameter and for three values of $B_{\text {ambient }}$ in formula (1). The $k$ values equal to $2,2.5$ and 3 were tested with $B_{\text {ambient }}=12 \mathrm{nT}$ (Fig. 4a). We also considered the cases where $k$ was equal to $2.5, B_{\text {ambient }}=0.12,1.2$ and $12 \mathrm{nT}$ (Fig. $4 \mathrm{~b}$ ), and $B_{\text {ambient }}=12, B_{\text {crust, } 100 \mathrm{~km}}=0$ (no CMF), 23 (weak CMF), and 150 (moderate CMF) nT in the approximation (1) of the crustal magnetic field (Fig. 4c). The distribution function of the precipitating electrons was assumed Maxwellian with $E_{0}=100 \mathrm{eV}$ and a downward energy flux of $1 \mathrm{~mW} \mathrm{~m}^{-2}$.

The upper panel of Fig. 4a shows the calculated height profiles of the downward (solid lines) and upward (dashed lines) energy fluxes. The results are shown for three different altitude dependences of the magnetic field: $k=3$ (red line), $k=2.5$ (black line) and $k=2$ (blue line). Both the upward and downward electron fluxes decrease with altitude, but their ratio varies with height. They become negligible below $120 \mathrm{~km}$ where the residual flux of precipitated electrons becomes increasingly small as a consequence of its loss of energy in the elastic and inelastic collisions. Changing parameter $k$, which controls the altitude dependence of the magnetic field magnitude, leads to some flux changes. A larger
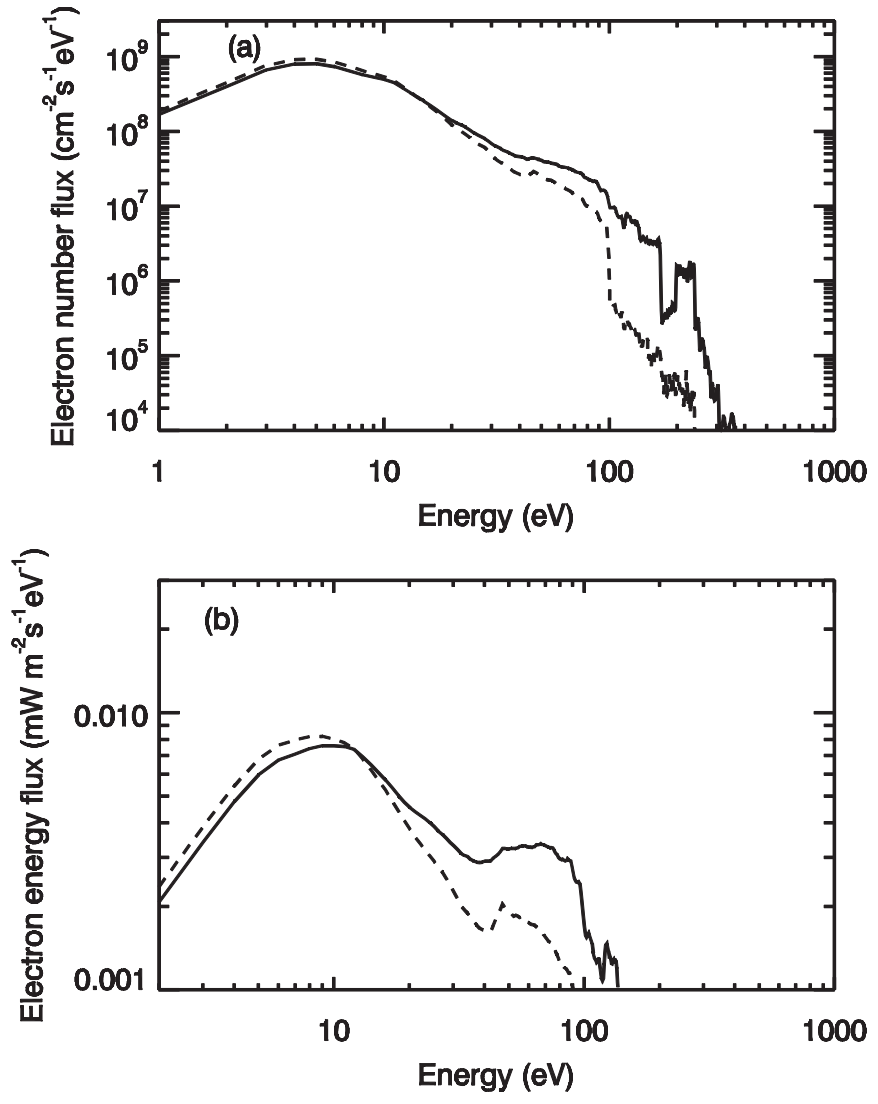

Fig. 2. Electron (a) number and (b) energy upward fluxes at $286 \mathrm{~km}$. Electron fluxes calculated with and without (dashed lines) the crustal magnetic field. These runs were conducted using the electron flux from ASPERA-3 measurements (10 May 2010 at 07:43) as an input at upper boundary.

$k$ value, which corresponds to a faster decrease of the intensity of $B$, leads to the highest fluxes, as may be expected from the decreasing effect of magnetic mirroring at higher altitude. However, the variation is relatively small. The bottom panel shows the height profiles for the ratio of upward and downward energy fluxes and its dependence on parameter $k$. This ratio varies with altitude between $\sim 0.5$ at $120 \mathrm{~km}$ and $\sim 0.3$ at $286 \mathrm{~km}$, with a dip near $150 \mathrm{~km}$, just above the maximum energy deposition at $140 \mathrm{~km}$. The calculated variations for different $k$ values are in the range of a few percent near $150 \mathrm{~km}$. It can thus be argued that our quantitative estimates of the influence of the Martian magnetic field on the aurora electron flux and UV emission are quite reliable and are suitable for various $k$ parameters in formula (1).

The upper panel of the Fig. $4 \mathrm{~b}$ shows the calculated height profiles of the downward (solid lines) and upward (dashed lines) energy fluxes for the sensitivity runs for the topology of the magnetic field with $k=2.5$ and $B_{\text {ambient }}=0.12,1.2$ and $12 \mathrm{nT}$ in the approximation (1) of the crustal magnetic field. The bottom panel shows the height profiles for the ratio of upward and downward energy fluxes. When the ambient component of the magnetic field in the approximation (1) is small the height-dependent second term in (1) is more efficient in the formation of the backscattered flux of the precipitating electrons due to magnetic mirroring. The ratio of upward and downward fluxes at the top of the model atmosphere can be as high as $\sim 0.8$ for the case of $B_{\text {ambient }}=0.12$.

The role of the crustal magnetic field (CMF) can be appreciated from the results presented in Fig. 4c which shows the same dependences as in Fig. 4a for the sensitivity runs in which the following topology of the magnetic field was used: $k=2.5, B_{\text {ambient }}=12$, and $B_{\text {crust, }} 100 \mathrm{~km}=0$ (no CMF), 23 (weak CMF), and 150 (moderate CMF) 


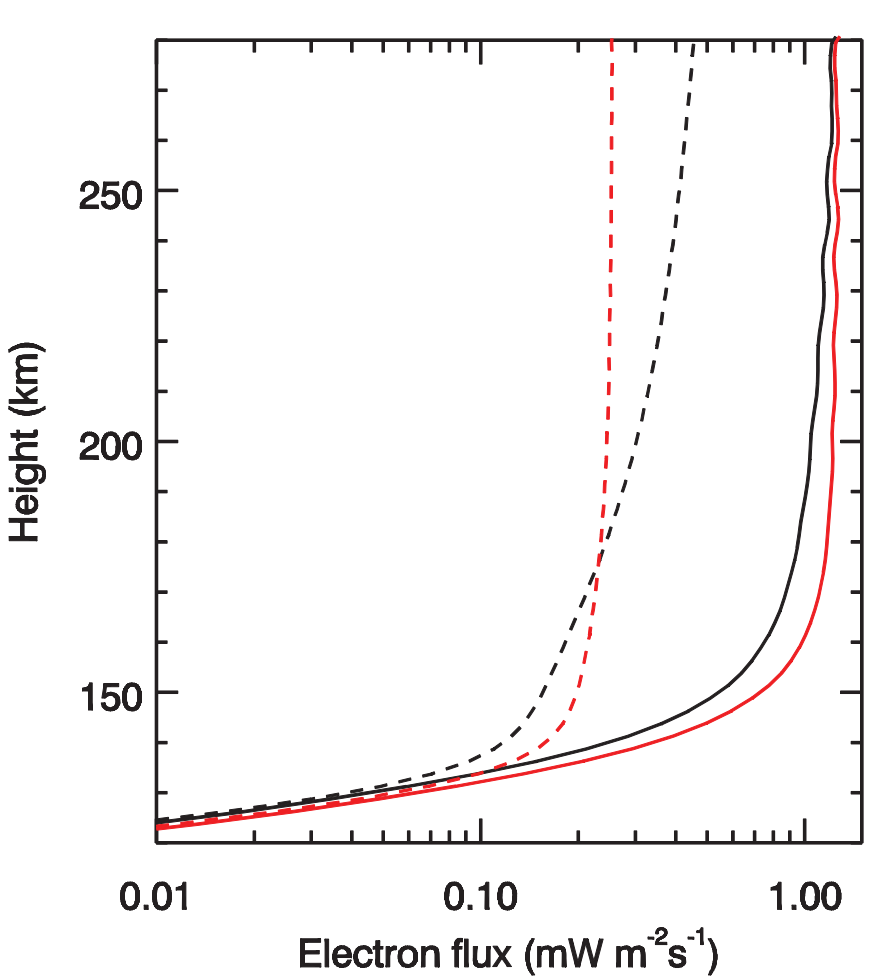

Fig. 3. Height profiles of the downward (solid line) and upward (dashed line) energy fluxes for a magnetic field characterized by $k=2.5, B_{\text {ambient }}=12$, and $B_{\text {crust, } 100 \mathrm{~km}}=0$ (without CMF; red line), and 23 (with weak CMF; black line) in approximation (1) of the CMF. These runs were conducted using the electron flux from ASPERA-3 measurements as an input at upper boundary. (For interpretation of the references to colour in this figure legend, the reader is referred to the web version of this article.)
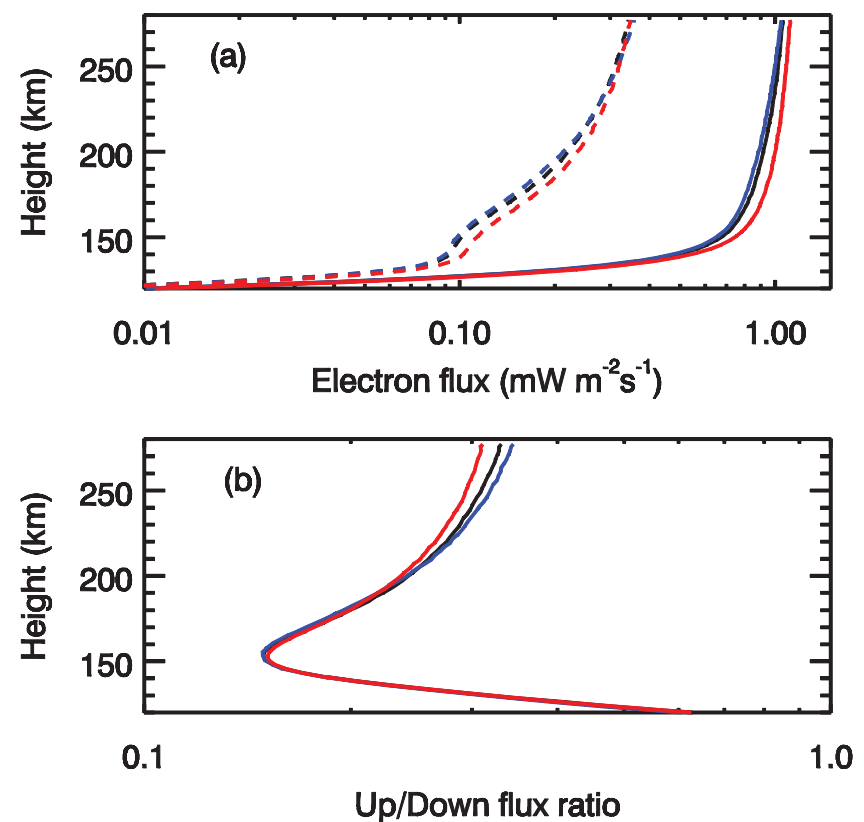

Fig. 4a. (a): Height profiles of downward (solid lines) and upward (dashed lines) energy fluxes for three different geometries of the magnetic field: the red line corresponds to $k=3$, black line to $k=2.5$, and blue line to $k=2$. $B_{\text {ambient }}$ in approximation (1) of the crustal magnetic field was taken equal to $12 \mathrm{nT}$, and $B_{\text {crust, } 100 \mathrm{~km}}=23$ nT. (b): Height profiles of the ratio of upward and downward energy fluxes for the same three cases. (For interpretation of the references to colour in text, the reader is referred to the web version of this article.)
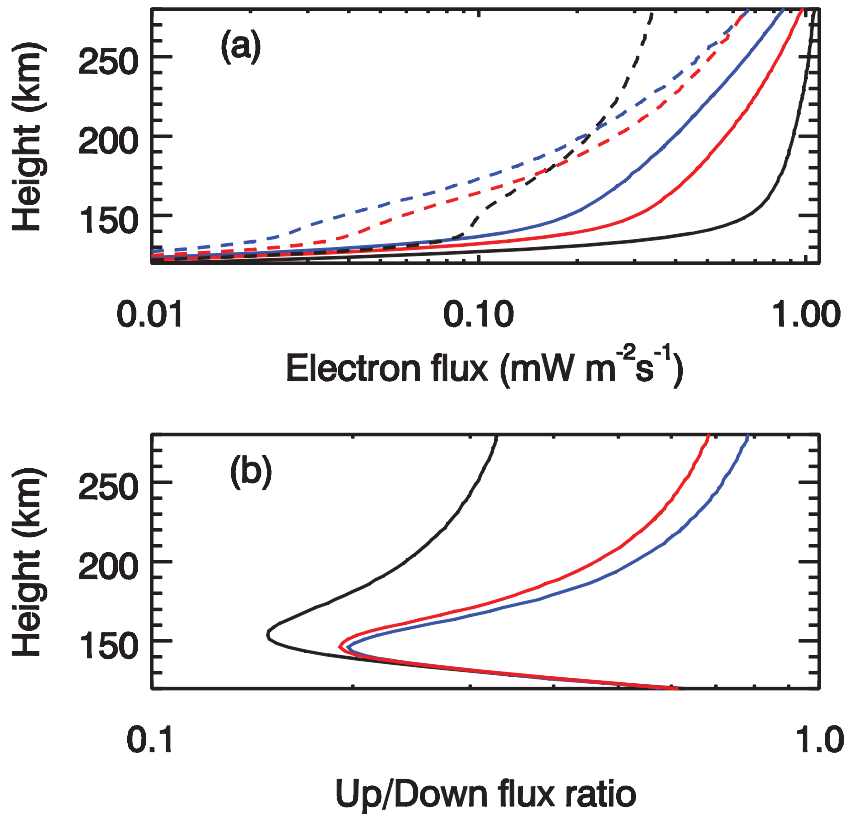

Fig. 4b. (a): Height profiles of the downward (solid line) and upward (dashed line) energy fluxes for the geometry of the magnetic field with $k=2.5, B_{\text {crust, } 100 \mathrm{~km}}=23$, and $B_{\text {ambient }}=0.12$ (blue line), 1.2 (red line) and 12 (black line) $\mathrm{nT}$ in approximation (1) of the crustal magnetic field. (b): Height profiles for the ratio of upward and downward energy fluxes for the same three cases. (For interpretation of the references to colour in this figure legend, the reader is referred to the web version of this article.)

nT in the approximation (1) of the crustal magnetic field. Upper panel of Fig. 4c shows the calculated height profiles of the downward (solid lines) and upward (dashed lines) energy fluxes. The bottom panel shows the height profiles for the ratio of upward and downward energy fluxes. This ratio approached 0.1 (no CMF), 0.3 (weak CMF), and 0.6 (moderate CMF) at the upper boundary of the model, with a dip near $150-170 \mathrm{~km}$ depending on the value of CMF.

As it is seen in Fig. $4 a-c$ the ratio between the upward and downward energy flux computed at the top of the atmosphere $(286 \mathrm{~km})$ is very sensitive to the presence of the magnetic field. Indeed, for the case without crustal magnetic field the flux ratio is about 0.1 , while inclusion of the magnetic field drastically increases this ratio as a result of mirroring. For typical geometry of the magnetic field the flux ratio at $286 \mathrm{~km}$ can be as large as 0.3 (see Fig. 4a), i.e. three times larger than in the non-magnetic case. It is important to note that this solution is weakly sensitive to the value of parameter $k$ in formula (1). It is found that parameters $B_{\text {ambient }}$ and $B_{\text {crust, } 100 \mathrm{~km}}$ in formula (1) have a much larger influence on the solution. At the top of the atmosphere the upward and downward energy flux ratio increases from $\sim 0.3$ up to $\sim 0.8$ (see Fig. $4 \mathrm{~b}$ ) when decreasing $B_{\text {ambient }}$ from 12 to $0.12 \mathrm{nT}$, and increases from 0.1 up to $0.3-0.5$ when CMF is switched off $\left(B_{\text {crust, }} 100 \mathrm{~km}=0 \mathrm{nT}\right)$ or it has weak $\left(B_{\text {crust, }} 100 \mathrm{~km}=23 \mathrm{nT}\right)$ or moderate $\left(B_{\text {crust, }} 100 \mathrm{~km}=150 \mathrm{nT}\right)$ values. We note that by changing the characteristics of the magnetic field (lower values of $B_{\text {ambient }}$ and/or stronger magnitudes of $B_{\text {crust, } 100 \mathrm{~km}}$ of CMF) it is possible to approach the low values $\sim 16 \%$ of the deposition of downward electron flux inferred from observations made on board the Mars Global Surveyor spacecraft (Shane et al., 2016).

Another factor that influences the electron fluxes at the top of the model is the energy of the precipitating electrons. To understand how energy controls the resulting upward flux, we conducted a series of simulations with monoenergetic input flux, the characteristic energy of which was $E_{0}=25,50,100,200,400$, 
Table 1a

Role of the Martian crustal magnetic field (CMF) on the electron energy flux at the top of the model $-\mathrm{R}=$ upward/downward energy flux ratio in [\%] in the case of an initial quasi-isotropic PAD.

\begin{tabular}{lllllllll}
\hline $\mathrm{E}_{0}(\mathrm{eV})$ & 25 & 50 & 100 & 200 & 400 & 600 & 800 & 1000 \\
\hline $\mathrm{R}$ - without CMF & 29.8 & 28.5 & 22.1 & 13.9 & 11.9 & 10.8 & 10.8 & 10.8 \\
$\mathrm{R}$ - with CMF & 44.1 & 43.4 & 40.9 & 32.8 & 33.4 & 35.0 & 35.9 & 37.1 \\
\hline
\end{tabular}
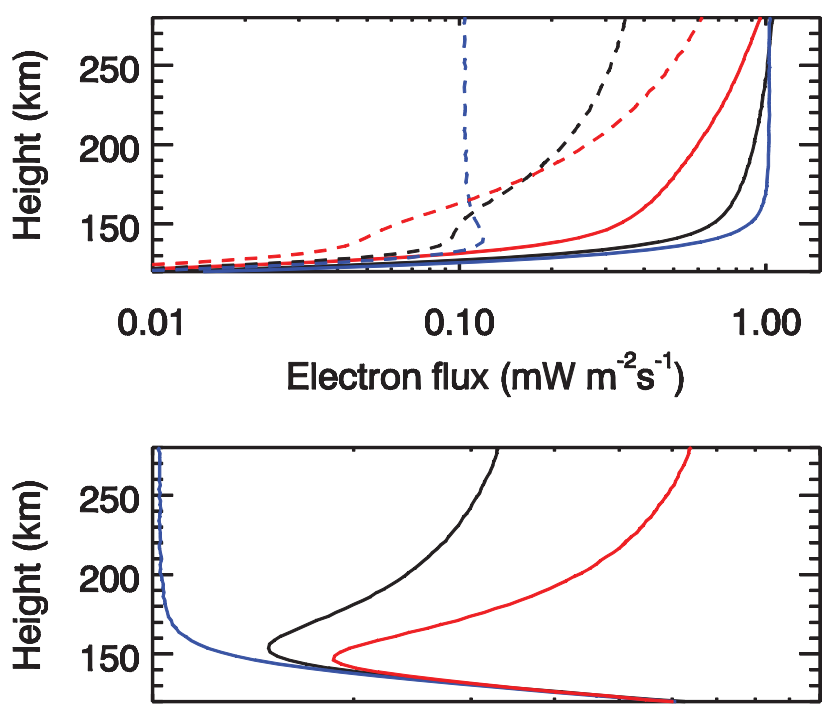

0.1 1.0

\section{Up/Down flux ratio}

Fig. 4c. (a): Height profiles of the downward (solid line) and upward (dashed line) energy fluxes for the geometry of the magnetic field with $k=2.5, B_{\text {ambient }}=12$, and $B_{\text {crust, } 100 \mathrm{~km}}=0$ (blue line), 23 (black line) and 150 (red line) nT in approximation (1) of the crustal magnetic field.(b): Height profiles for the ratio of upward and downward energy fluxes for the same three cases. (For interpretation of the references to colour in this figure legend, the reader is referred to the web version of this article.)

\section{Table 1b}

Role of the Martian crustal magnetic field (CMF) on the electron energy flux at the top of the model $-\mathrm{R}=$ upward/downward energy flux ratio in [\%] in the case of an initial field-aligned PAD.

\begin{tabular}{lcccccccc}
\hline $\mathrm{E}_{0}(\mathrm{eV})$ & 25 & 50 & 100 & 200 & 400 & 600 & 800 & 1000 \\
\hline $\mathrm{R}$ - without CMF & 17.6 & 17.3 & 10.9 & 4.1 & 2.2 & 1.4 & 1.2 & 1.1 \\
$\mathrm{R}$ - with CMF & 18.1 & 17.5 & 12.4 & 4.5 & 2.5 & 1.6 & 1.2 & 1.1 \\
\hline
\end{tabular}

$600,800,1000 \mathrm{eV}$, respectively. The initial pitch angle distribution (PAD) is assumed to be quasi-isotropic (Table 1a) or field-aligned (Table $1 \mathrm{~b}$ ), i.e., when precipitating electrons are directed strictly along the magnetic field line. The ratio between the computed upward and downward energy fluxes for a model with typical geometry of the magnetic field $\left(k=2.5, B_{\text {ambient }}=12 \mathrm{nT}\right.$, and $B_{\text {crust, }} 100 \mathrm{~km}=23$ ) and different PADs are presented in Tables $1 \mathrm{a}$ and $1 \mathrm{~b}$. In the simulations without crustal magnetic field, the energy flux ratio is relatively low and drops from $29.8 \%$ to $10.8 \%$ for initial energies increasing from 25 to $600 \mathrm{eV}$. This decrease of the ratio is a consequence of collisional angular scattering that is more effective at low initial electron energy, so that the collisions more efficiently repel the low energy particles back to the top of the simulation domain. The situation is quite different in the presence of a magnetic field for which the computed upward energy fluxes are significantly higher than without B-field. They vary from $33 \%$ up to $44 \%$. This difference is caused by the additional effect of magnetic mirroring that causes a substantial fraction of the electron beam to be reflected and returned back to space. A slight increase of the relative importance of the upward flux is predicted for higher electron energy. However, even for a 20 -fold increase of the electron energy, the upward flux remains above $30 \%$ of the downward flux and the change in flux ratio remains within $\sim 10 \%$.

In the case of initial field-aligned PAD of the precipitating electrons the situation is quite different. It is seen in Table $1 \mathrm{~b}$ that the computed upward energy fluxes in the presence of a crustal magnetic field (1) are significantly lower than ones for quasi-isotropic PAD. This is caused by the fast penetration of the monoenergetic flux with field-aligned PAD to the heights below $140 \mathrm{~km}$, where high-energy electrons locally deposit their kinetic energy to the ambient atmospheric gas and only a small fraction of electrons are reflected following collisions with neutrals and returned back to space.

\subsection{Effects on auroral emissions}

Let us consider the influence of the CMF on the auroral emissions. As a population of electrons spirals down toward the Martian atmosphere, the intensity of the crustal field increases and the area of flux tube decreases by a factor $B(z) / B\left(z_{0}\right)$, where $z_{0}$ is the altitude of the top of the model. Accordingly, the electron flux (per unit area) increases by the same factor. Therefore, flux values calculated by the one-dimensional Monte Carlo model should also be multiplied in the same proportion. Conservation of the first adiabatic invariant acts in the opposite direction as the electrons with large pitch angles mirror back as they move downward. In the absence of angular scattering by the neutral constituents and for an initially isotropic pitch angle distribution, the two effects cancel out and the electron flux remains invariant along the flux tube. It is important to note that this cancellation only applies to the total electron flux, i.e. the omnidirectional number of electrons crossing a unit area. This has the consequence that volume emission rates will only slightly respond to the presence of a magnetic field. A different aspect is the response in terms of emitted auroral power when considering a given spatially confined region where a given input power is carried by precipitating auroral electrons. The emitted auroral power is the emission integrated over the size of the associated aurora. Although the computed local flux is only weakly modified by the inclusion of the magnetic field and of the mirroring process, the power is not left unchanged by the inclusion of the field because it has the effect of reducing the effective area over which the emission must be integrated, as a consequence of the decrease of the flux tube area for decreasing z. In this case, the loss of the mirroring population is the dominant effect: mirrored particles return to space without depositing their energy in the atmosphere.

To test these statements, we have conducted calculations of two emissions in the Mars atmosphere. The first one is the CO Cameron bands corresponding to the $\mathrm{a}^{3} \Pi \rightarrow \mathrm{X}^{1} \Sigma$ transition. They have been observed (Bertaux et al., 2005; Leblanc et al., 2008; Gérard et al., 2015; Soret et al., 2016) in the Martian aurora with a nadir intensity ranging from 210 to $1880 \mathrm{R}$ in the wide section part of the SPICAM slit (the SPICAM design included a double-shaped slit with two different values of the slit width). The emission threshold 


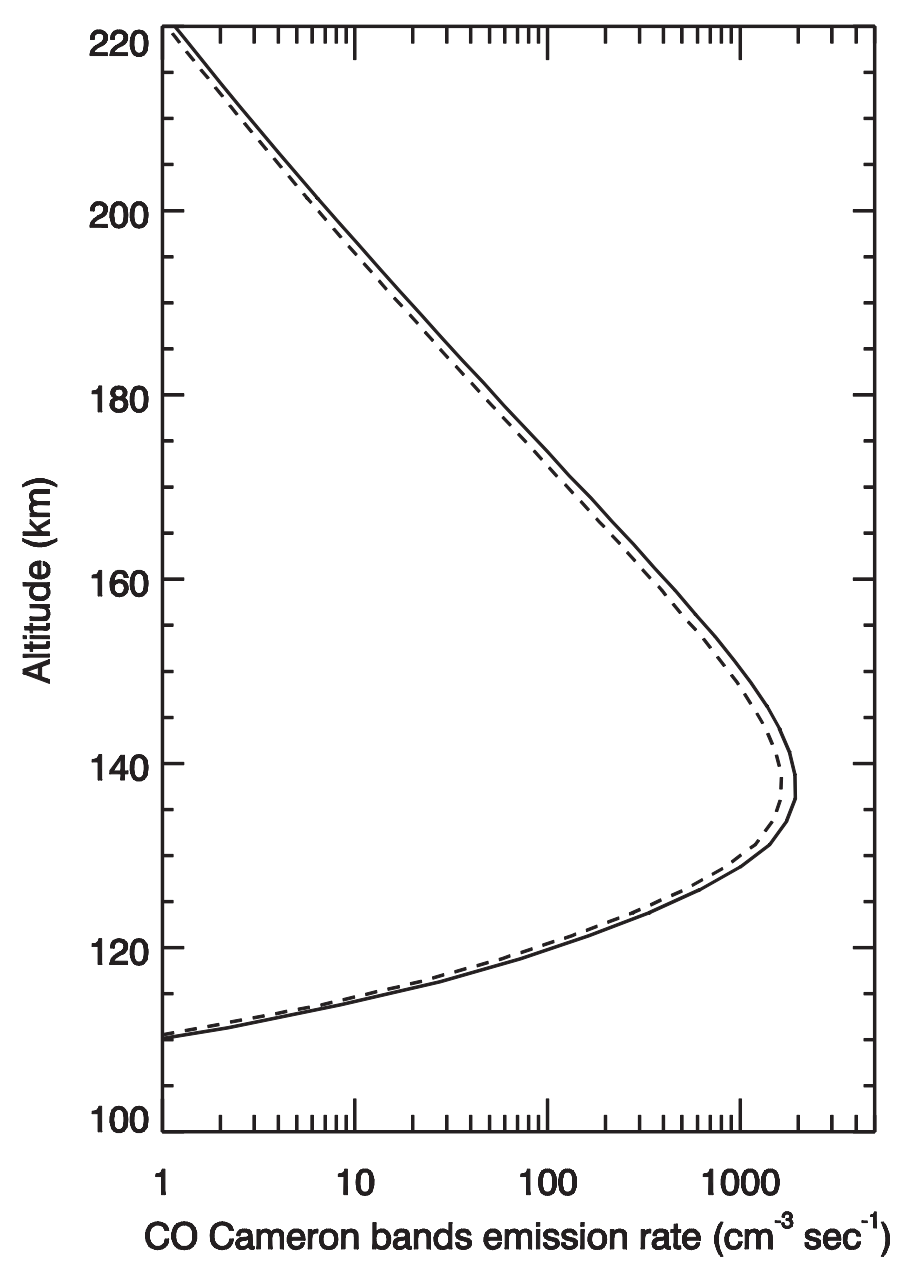

Fig. 5. Height profiles of Cameron band emission rate for runs with (solid line) and without (dashed line) magnetic field.

is at $11.9 \mathrm{eV}$ and the cross section for electron impact peaks near $80 \mathrm{eV}$ The second one is the transition that emits the $\mathrm{CO}_{2}{ }^{+}$FoxDuffendack-Barker (FDB) bands from 300 to $450 \mathrm{~nm}$ and is probably the emission that would be visible to the naked eye from the Martian surface. The effect of the magnetic field is shown for the $\mathrm{CO}$ Cameron (Fig. 5) and $\mathrm{CO}_{2}{ }^{+}$FDB bands (Fig. 6), for cases with (solid line) and without (dashed line) crustal magnetic field. Calculations were conducted using the electron flux from ASPERA-3 measurements (10 May 2010 at 07:43) and initial isotropic PAD as the model inputs in both runs with and without residual magnetic field. In all calculations with the crustal magnetic field a reference altitude $z_{0}=286 \mathrm{~km}$ was used for the $B(\mathrm{z}) / B\left(\mathrm{z}_{0}\right)$ factor.

The analysis of the vertical profiles of the Cameron bands shows only minor changes in the emission rate profile. There is also a slight change in the altitude of the peak: in the presence of a crustal magnetic field the maximum of the emission is found $\sim 5 \mathrm{~km}$ higher than without. The column brightness of the Cameron bands is $4.8 \mathrm{kR}$ and $4.4 \mathrm{kR}$ with and without the magnetic field respectively. It is worth noting that initial PAD of precipitating electrons plays an important role. If we consider the isotropic PAD (in sense of random selection of angle $\theta$ ) the column brightness decreases and equal to $\sim 4.7 \mathrm{kR}$. For the field-aligned initial PAD it reaches the value of $\sim 4.9 \mathrm{kR}$ as a consequence of the reduction of the upward flux.

Calculations of the $\mathrm{CO}_{2}+\mathrm{FDB}$ volume emission rate (Fig. 6) show a non-significant change in emission rate. At the emission peak the reduction is approximately the same, and the peak height

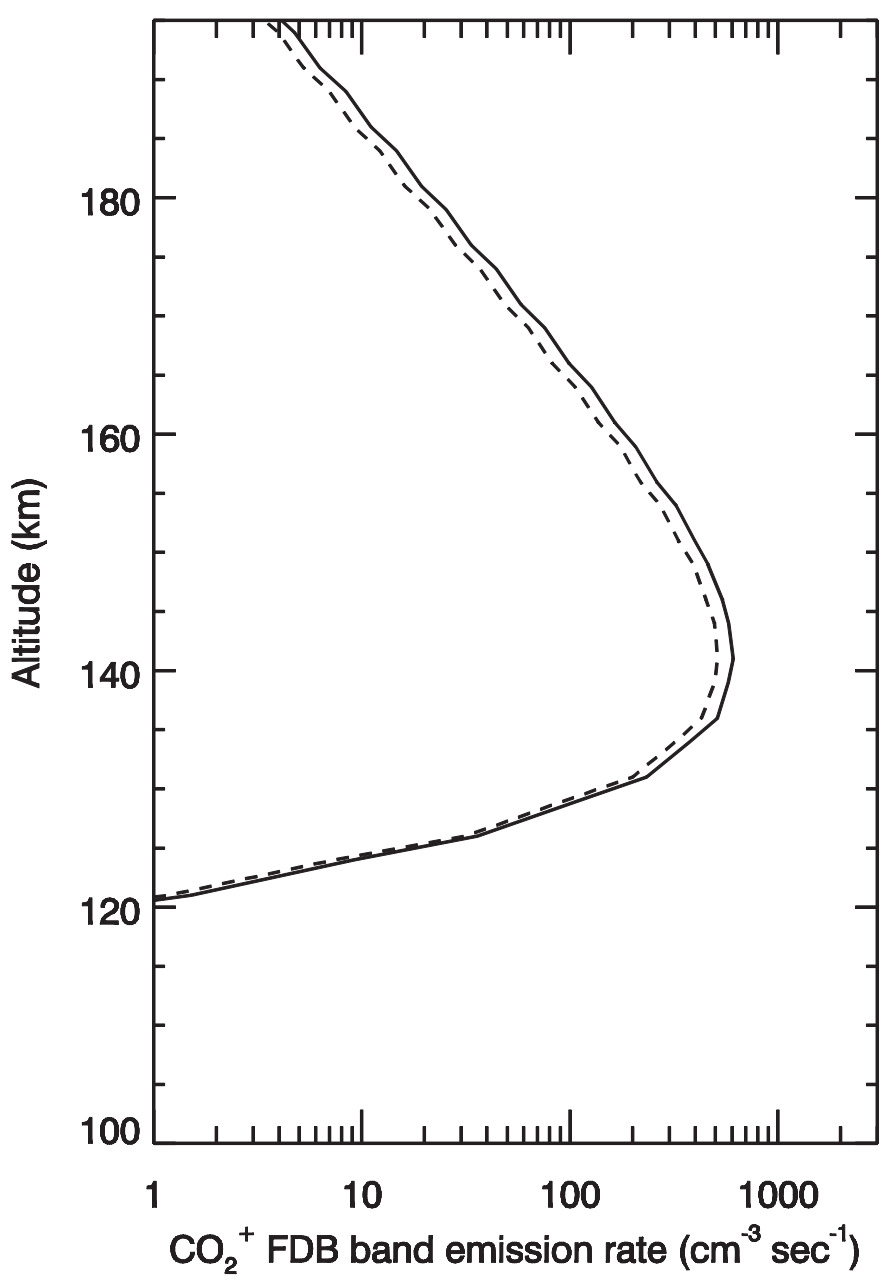

Fig. 6. Height profiles of $\mathrm{CO}_{2}+\mathrm{FDB}$ band emission rate for runs with (solid line) and without (dashed line) magnetic field.

does not change. The column brightness of the $\mathrm{CO}_{2}{ }^{+} \mathrm{FDB}$ bands increases from $1.3 \mathrm{kR}$ for the case without crustal magnetic field up to $1.5 \mathrm{kR}$ in the solution with the crustal magnetic field, and is not strongly dependent on the initial PAD.

As it was mentioned before, another factor that influences the electron flux at the top of the model is the energy of the precipitating electrons. To understand how energy controls the excitation rates of the auroral emissions, we conducted a series of simulations with a monoenergetic input flux, the characteristic energy of which (noted $E_{0}$ ) was changed from $25 \mathrm{eV}$ up to $1000 \mathrm{eV}$ The initial pitch angle distributions (PAD) were assumed to be either quasiisotropic or field-aligned.

The influence of the energy of the precipitating electrons on the luminosity correlates well with the solutions without magnetic field (see, e.g., Soret et al., 2016). Indeed, as presented in Fig. 7, the vertical profiles of the Cameron bands emission rate, calculated for the case with magnetic field and isotropic PAD, show that the decrease of the energy leads to a significant increase of the altitude of the peak of the emission rate, while the total emissivity varies within $30 \%$ only (the total energy flux of precipitating electrons in the model was the same for all runs). The calculated column brightness in aurora is a factor of $\sim 1.25$ higher in the presence of the crustal magnetic field. However, the crustal magnetic field results in increase of the upward electron flux (see Tables 1a and $1 \mathrm{~b})$, and, consequently, in reduction of the total auroral brightness (or emitted auroral power) for given energy flux of precipitating electrons. 


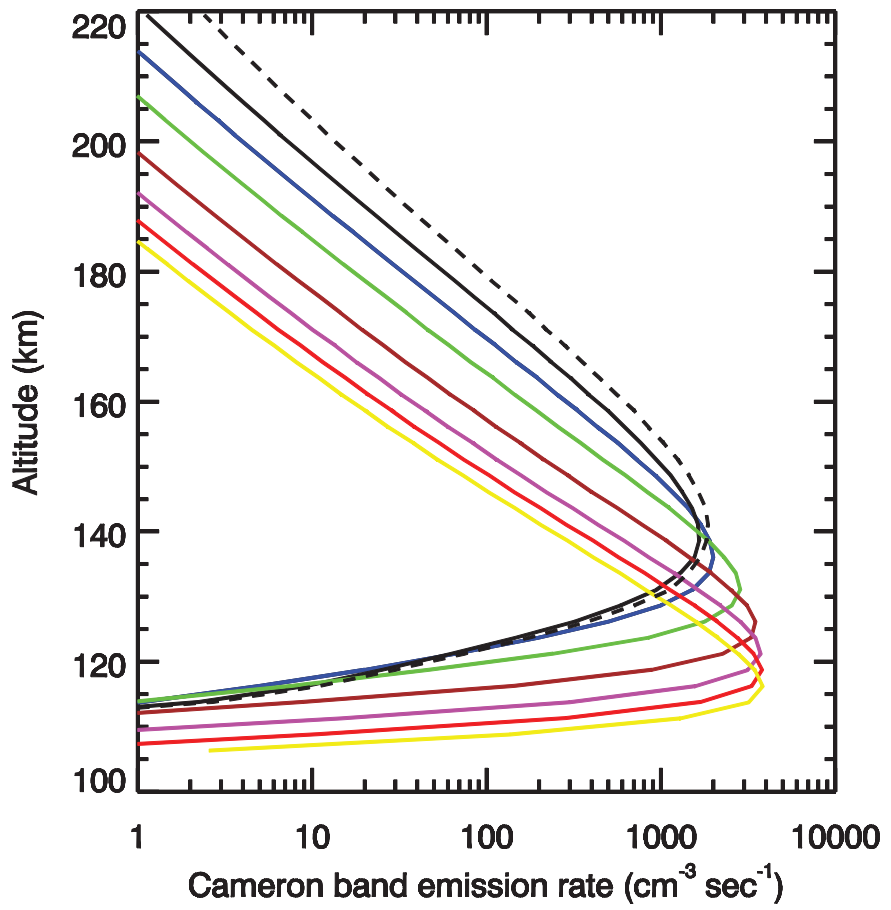

Fig. 7. Height profiles of Cameron bands for monoenergetic electron precipitation of $1 \mathrm{~mW} \mathrm{~m}^{-2}$ with $\mathrm{E}_{0}=50,100,200,400,600,800$, and $1000 \mathrm{eV}$ (from black to yellow). The case $\mathrm{E}_{0}=25 \mathrm{eV}$ is shown by black dashed line. (For interpretation of the references to colour in this figure legend, the reader is referred to the web version of this article.)

\section{Conclusions}

We have conducted simulations of the auroral precipitation at Mars using a Monte Carlo model, accounting for the crustal magnetic field and of a possible ambient constant field. The auroral emissions of the $\mathrm{CO}$ Cameron and $\mathrm{CO}_{2}+\mathrm{FDB}$ bands have also been computed. The analysis of the sensitivity of the calculations versus the control parameters of the model shows that the vertical dependence of the magnetic field and the energy of the precipitating electrons both influence the solution.

(1) The ratio between the upward and downward energy fluxes is very sensitive to the presence of the magnetic field. Indeed, for the case without crustal magnetic field the calculated ratio at the top of the model is $\sim 0.1$ (see, Fig. $4 \mathrm{c}$ ), while inclusion of the crustal magnetic field drastically increases this ratio under the effect of magnetic mirroring. For typical topology of the magnetic field the flux ratio at the top could be as large as 0.3 (see Fig. 4a and c), and even 0.6 in the case of moderate value of the crustal magnetic field. It is important to note that this solution is only weakly sensitive to the adopted altitude dependence of the field represented by parameter $k$ in formula (1). It is found that $B_{\text {ambient }}$ parameter in formula (1) exerts a much stronger control as demonstrated by the ratio between upward and downward energy fluxes increases from 0.3 up to 0.8 when decreasing the $B_{\text {ambient }}$ from 12 to $0.12 \mathrm{nT}$.

(2) The energy of the precipitating electrons is another important quantity that influences the auroral flux at the top of the model. To understand how energy controls the resulting upward flux, a set of runs with monoenergetic input fluxes $\mathrm{E}_{0}=25-1000 \mathrm{eV}$ was conducted for a model with typical topology of the magnetic field $\left(k=2.5\right.$ and $\left.B_{\text {ambient }}=12 \mathrm{nT}\right)$ and different initial pitch angle distributions assumed to be either isotropic or field-aligned. In the simulations without magnetic field the energy flux ratio is relatively low and drops from $30 \%$ to $11 \%$ for initial energies increasing from 25 to $600 \mathrm{eV}$ This decrease of the ratio is a consequence of collisional angular scattering that is more effective at low initial electron energy. Consequently collisions more efficiently backscatter the low energy electrons to the top of the simulation domain. The situation is quite different in the presence of a crustal magnetic field for which the upward energy fluxes are significantly higher than in the absence of a B-field. They vary from $33 \%$ up to $44 \%$. A slight increase of the relative importance of the upward flux is predicted for higher electron energy. However, even for a 20 -fold increase of the electron energy, the upward flux remains above $30 \%$ of the downward flux and the change in flux ratio remains within $\sim 10 \%$.

(3) The auroral column brightness computed assuming an isotropic precipitation is only weakly increased by the presence of the magnetic field, due to the competing effect of mirroring and field line convergence. In contrast, the auroral power is reduced by the presence of the magnetic field as part of the precipitating energy is mirrored back to space.

(4) To relate these model results with in situ measurements, we used an electron energy spectrum of precipitating electrons obtained by the ASPERA-3 on board Mars Express. For the simulations we used the energy distribution function of electrons, measured on 10 May 2010 at 07:44 UT at the altitude of $590 \mathrm{~km}$. The energy flux strongly decreases above $200 \mathrm{eV}$, reaching a very low value at $300 \mathrm{eV}$. The peak energy is $90 \mathrm{eV}$ and the mean electron energy is $\sim 140 \mathrm{eV}$ (Gérard et al., 2015). The simulation results obtained in this case indicate that the upward energy flux can be as large as $33 \%$ of the downward one. It is important to note that the low-energy core of the upward flux is formed by collisional spreading. In contrast, the upward flux at high energies mostly results from magnetic mirroring. To discriminate between the contributions of magnetic mirroring and collisional scattering to the formation of the upward flux, we compared two simulations made keeping all model parameters identical, one with and one without the effect of a crustal magnetic field. The second one leads to a significant decrease, up to $20 \%$, in the upward energy flux. Thus, the presence of the crustal magnetic field leads to a marked, energy-dependent, increase of the upward electron energy flux from $20 \%$ to $33 \%$.

Finally, this study shows that even a weak magnetic field can strongly change the effects of the high-energy electron precipitation in planetary atmospheres such as energy deposition, ionization and excitation rates and etc. Therefore, even weak magnetic fields should be taken into account when the heating efficiency of the hydrogen- and water vapor atmospheres of the exoplanets are considered (Bisikalo and Shematovich, 2015).

(5) However, the difference between the solutions remains within a factor of $1.5-2$, when changing the model parameters within the range of values acceptable for Mars. These conclusions may be generalized and are applicable to estimate the influence of the residual crustal magnetic field on the electron flux and UV brightness in the Martian aurora, and to the interpretation of a wide range of existing and future measurements.

\section{Acknowledgments}

D.B. and V.S. acknowledge financial support by the Russian Science Foundation (Project nr. 14-12-01048). BH is supported by the Belgian Fund for Scientific Research. This research was partly 
funded by the PRODEX CODYMAV program managed by the European Space Agency with help of the Belgian Science Policy Office (BELSPO) and by BELSPO' SCOOP/BRAIN program.

\section{References}

Acuña, M.H., 19 colleagues, 1998. Magnetic field and plasma observations at Mars: Initial results of the Mars Global Surveyor mission. Science 279, 1676-1680. http://dx.doi.org/10.1126/science.279.5357.1676.

Ajello, J.M., 1971. Emission cross sections of $\mathrm{CO}_{2}$ by electron impact in the interval 1260-4500 A. II.. J. Chem. Phys. 55, 3169-3177. http://dx.doi.org/10.1063/1. 1676564.

Avakyan, S.V., et al., 1998. Collision Processes and Excitation of Ultraviolet Emission from Planetary Atmospheric Gases: A Handbook of Cross Sections. Gordon and Breach, London.

Barabash, S., et al., 2006. The analyzer of space plasmas and energetic atoms (ASPERA-3) for the Mars express mission. Space Sci. Rev. 126, 113-164. http: //dx.doi.org/10.1007/s11214-006-9124-8.

Bertaux, J.-L., Leblanc, F., Witasse, O., Quemerais, E., Lilensten, J., Stern, S.A Sandel, B., Korablev, O., 2005. Discovery of an aurora on Mars. Nature 435, 790794. http://dx.doi.org/10.1038/nature03603.

Bisikalo, D.V., Shematovich, V.I., 2015. Precipitation of electrons into the upper atmosphere of a hot-jupiter exoplanet. Astron. Rep. 59, 836-842. http://dx.doi. org/10.1134/S1063772915090024.

Bittencourt, J.A., 2004. Fundamentals of Plasma Physics. third ed. by J.A. Bittencourt Springer-Verlag, New York.

Bougher, S.W., Pawlowski, D., Bell, J.M., Nelli, S., McDunn, T., Murphy, J.R., Chizek, M. Ridley, A., 2015. Mars global ionosphere-thermosphere model (MGITM): Solar cycle, seasonal, and diurnal variations of the Mars upper atmosphere. J. Geophys. Res. 120. http://dx.doi.org/10.1002/2014JE004715.

Brain, D.A., Halekas, J.S., Peticolas, L.M., Lin, R.P., Luhmann, J.G., Mitchell, D.L. Delory, G.T., Bougher, S.W., Acuña, M.H., Rème, H, 2006. On the origin of aurorae on Mars. Geophys. Res. Lett. 33, L01201. http://dx.doi.org/10.1029/ 2005GL024782.

Brain, D.A., Lillis, R.J., Mitchell, D.L., Halekas, J.S., Lin, R.P., 2007. Electron pitch angle distributions as indicators of magnetic field topology near Mars. J. Geophys. Res. 112, A09201. http://dx.doi.org/10.1029/2007JA012435.

Decker, D.T., Kozelov, B.V., Basu, B., Jasperse, J.R., Ivanov, V.E., 1996. Collisional degradation of the proton- $\mathrm{H}$ atom fluxes in the atmosphere: a comparison of theoretical techniques. J. Geophys. Res. 101, 26947-26960. http://dx.doi.org/10.1029/ 96JA02679

Erdman, P.W., Zipf, E.C., 1983. Electron-impact excitation of the cameron system $($ a3P $\rightarrow$ X1S) transition of CO. Planet. Space Sci. 31, 317-321. http://dx.doi.org 10.1029/JA090iA11p11087.

Ferguson, B.B., Cain, J.C., Crider, D.H., Brain, D.A., Harnett, E.M., 2005. External fields on the nightside of Mars at Mars global surveyor mapping altitudes. Geophys. Res. Lett. 32, L16105. http://dx.doi.org/10.1029/2004GL021964.

Gérard, J.-C., Hubert, B., Shematovich, V.I., Bisikalo, D.V., Gladstone, G.R., 2008. The Venus ultraviolet oxygen dayglow and aurora: model comparison with observations. Planet. Space Sci. 56, 542-552. http://dx.doi.org/10.1016/j.pss.2007.11.008.

Gérard, J.-C., Soret, L., Libert, L., Lundin, R., Stiepen, A., Radioti, A., Bertaux, J.-L. 2015. Concurrent observations of ultraviolet aurora and energetic electron precipitation with Mars express. J. Geophys. Res. 120. http://dx.doi.org/10.1002/ 2015JA021150.

Gilijamse, J.J., et al., 2007. The radiative lifetime of metastable CO (a3P, $n=0$ ). 221102-1-221102-4. J. Chem. Phys. 127. 221102-1-221102-4 http://dx.doi.org/ $10.1063 / 1.2813888$
Gronoff, G., Simon Wedlund, C., Mertens, C.J., Barthélemy, M., Lillis, R.J., Witasse, O., 2012. Computing uncertainties in ionosphere-airglow models: II. the martian airglow.. J. Geophys. Res. 117, A05309. http://dx.doi.org/10.1029/2011JA017308.

Halekas, J.S., Brain, D.A., Lin, R.P., Luhmann, J.G., Mitchell, D.L., 2008. Distribution and variability of accelerated electrons at Mars. Adv. Space Res. 1347-1352. http://dx.doi.org/10.1016/j.asr.2007.01.034.

Leblanc, F., et al., 2008. Observations of aurorae by SPICAM ultraviolet spectrograph on board Mars Express: simultaneous ASPERA-3 and MARSIS measurements. J. Geophys. Res. 113, A08311. http://dx.doi.org/10.1029/2008JA013033.

Lillis, R.J., Brain, D.A., 2013. Nightside electron precipitation at Mars: geographic variability and dependence on solar wind conditions. J. Geophys. Res. 118, 35463556. http://dx.doi.org/10.1002/jgra.50171.

Lillis, R.J., Fang, X., 2015. Electron impact ionization in the martian atmosphere: interplay between scattering and crustal magnetic field effects. J. Geophys. Res. Planets 120, 1332-1345. http://dx.doi.org/10.1002/2015JE004841.

Lillis, R.J., Fillingim, M.O., Peticolas, L.M., Brain, D.A., Lin, R.P., Bougher, S.W., 2009 Nightside ionosphere of Mars: modeling the effects of crustal magnetic fields and electron pitch angle distributions on electron impact ionization. J. Geophys. Res. 114, E11009. http://dx.doi.org/10.1029/2009JE003379.

Lillis, R.J., Fillingim, M.O., Brain, D.A., 2011. Three-dimensional structure of the martian nightside ionosphere: predicted rates of impact ionization from mars global surveyor magnetometer and electron reflectometer measurements of precipitating electrons. J. Geophys. Res. 116, A12317. http://dx.doi.org/10.1029/ 2011JA016982.

Lundin, R., et al., 2006a. Plasma acceleration above martian magnetic anomalies. Science 311, 980-983. http://dx.doi.org/10.1126/science.1122152.

Lundin, R., et al., 2006b. Ionospheric plasma acceleration at Mars: ASPERA-3 results. Icarus 182, 308-319. http://dx.doi.org/10.1016/j.icarus.2005.10.035.

Mitchell, D.L., Lin, R.P., Mazelle, C., Rème, H., Cloutier, P.A., Connerney, J.E.P., Acuna, M.H., Ness, N.F., 2001. Probing Mars' crustal magnetic field and ionosphere with the MGS electron reflectometer. J. Geophys. Res. 106 (23), 419-427. http://dx.doi.org/10.1029/2000JE001435.

Schneider, N.M., Deighan, J.I., Jain, S.K., Stiepen, A., Stewart, A.I.F., Larson, D., Mitchell, D.L., Mazelle, C., Lee, C.O., Lillis, R.J., Evans, J.S., Brain, D., Stevens, M.H., McClintock, W.E., Chaffin, M.S., Crismani, M., Holsclaw, G.M., Lefevre, F., Lo, D.Y. Clarke, J.T., Montmessin, F., Jakosky, B.M., 2015. Discovery of diffuse aurora on Mars. id. 0313. Science 350 (6261). id. 0313 http://dx.doi.org/10.1126/science. aad0313

Shane, A.D., Shaosui, X., Liemohn, M.W., Mitchell, D.W., 2016. Mars nightside electrons over strong crustal fields. J. Geophys. Res. Space Phys. 121, 3808-3823. http://dx.doi.org/10.1002/2015JA021947.

Shematovich, V.I., Bisikalo, D.V., Gérard, J.-C., Cox, C., Bougher, S.W., Leblanc, F., 2008. Monte carlo model of electron transport for the calculation of Mars dayglow emissions. J. Geophys. Res. 113, E02011. http://dx.doi.org/10.1029/ 2007JE002938.

Shematovich, V.I., Bisikalo, D.V., Diéval, C., Barabash, S., Stenberg, G., Nilsson, H. Futaana, Y., Holmstrom, M., Gérard, J.-C., 2011. Proton and hydrogen atom transport in the martian upper atmosphere with an induced magnetic field. J. Geophys. Res. 116, A11320. http://dx.doi.org/10.1029/2011JA017007.

Shirai, T., Tabata, T., Tawara, H., 2001. Analytic cross sections for electron collisions with $\mathrm{CO}, \mathrm{CO}_{2}$, and $\mathrm{H}_{2} \mathrm{O}$ relevant to edge plasma impurities. At. Data Nucl. Data Tables 79, 143-184.

Soret, L., Gérard, J.C., Libert, L., Shematovich, V.I., Bisikalo, D.V., Stiepen, A., Bertaux, J.L., 2016. SPICAM observations and modeling of Mars aurorae. Icarus 264, 398-406. http://dx.doi.org/10.1016/j.icarus.2015.09.023. 